\title{
NUEVOS ANTECEDENTES EN EL ESTUDIO PALINOLOGICO DE LOS MANTOS CARBONIFEROS DEL TERCIARIO DE ARAUCO-CONCEPCION, CHILE.
}

\author{
SYLVIA PALMA-HELDT \\ Departamento de Geología y Paleontología, Universidad de Concepción, Concepción, Chile
}

\section{RESUMEN}

Esta contribución da a conocer nuevos antecedentes que complementan los resultados expuestos como preliminares al I Congreso Latinoamericano de Paleontología en Abril de 1978.

En esta primera parte de un plan de estudio mucho más amplio del Terciario de Arauco-Concepción, el trabajo se ha limitado al análisis de cinco mantos carboníferos explotables del Miembro Lota, Formación Curanilahue.

Se presentan datos estadísticos basados en un número mayor de muestras que aquellas analizadas en la primera contribución. Esto ha permitido caracterizar los mantos y analizar matemáticamente la correlación existente entre ellos sobre la base del contenido polínico.

Se incluyen nuevos palinomorfos y sus respectivas descripciones. 


\section{ABSTRACT}

This paper continues the preliminary work presented at the I Congreso Latinoamericano de Paleontología in April, 1978.

In this first part of a much broader study of the Tertiary of Arauco-Concepción, the work has been circumscribed to the analysis of five exploited coal beds of the Lota Member, Curanilahue Formation.

Statistical data based on a greater number of samples than the previous paper are presented. This has allowed the characterization of the beds and the study of the mathematical correlation between them based on the palynological contents.

New palynomorphs and their descriptions are included.

\section{INTRODUCCION}

Tal como se mencionara en el trabajo presentado por la autora al I Congreso Latinoamericano de Paleontología en Abril de 1978, en la zona carbonífera del Terciario de AraucoConcepción se realiza una serie de investigaciones que son el fruto de un Convenio Universidad de Concepción - Empresa Nacional del Carbón (ENACAR). En esta primera etapa de un plan terciario mucho más amplio el trabajo se ha limitado al estudio palinológico de cinco mantos carboníferos del Miembro Lota, Formación Curanilahue. Se pretende obtener en un futuro próximo el registro de toda la secuencia terciaria de la zona.

$\mathrm{El}$ análisis de los datos paleopalinológicos obtenidos en estas primeras investigaciones se ha encausado con miras a obtener principalmente los siguientes objetivos:

- Contribuir al mejor conocimiento de la flora terciaria de Sudamérica.

- Aportar antecedentes a las interpretaciones paleogeográficas, paleoclimatológicas y paleoecológicas del Terciario Chileno.

- En un sentido más práctico, contar con una herramienta útil para la correlación en la zona de Arauco-Concepción.

- Por último, desde un punto de vista práctico y de aplicación inmediata, definir las diferencias y semejanzas en el contenido palinológico de los distintos mantos carboníferos del Miembro Lota. Obtener así un patrón estadístico de las formas que caracterizan a cada manto y poder llegar a determinar "muestras problemas" que se obtengan en el futuro.

El tratamiento matemático de la informa. ción es conducente a obtener este propósito.
La presente contribución se considera un complemento a los estudios palinológicos preliminares, se seguirá por lo tanto el mismo esquema de presentación utilizado en la primera contribución y en repetidas ocasiones se hará referencia a la información allí contenida.

En dicho trabajo se ilustra con el mapa respectivo la ubicación geográfica y el marco geológico del área en estudio. Así también se esquematiza la secuencia estratigráfica del Miembro Lota con la ubicación de los mantos carboniferos.

Se estima necesario repetir aquí que las muestras analizadas provienen del Miembro Lota, Formación Curanilahue, Eoceno inferior (Muffoz Cristi, 1968). El Miembro Lota es una secuencia continental constituída por areniscas de grano fino a grueso en parte conglomerádicas y conglomerados finos. En la secuencia se intercalan arcillolitas y los mantos de carbón, explotados en las minas de Lota y Schwager, ubicadas en la costa de la Provincia de Concepción, entre los 370 y los 37006' S.

Los mantos estudiados son los denominados de arriba hacia abajo: Manto 2 o Veta Nueva, Manto 3 o Veta Arriba, Manto 4 o Veta Chica, Manto 5 o Veta alta y Manto 7.

\section{ANALISIS PALINOLOGICO}

\section{a) Metodologia Empleada}

En este trabajo se presentan datos estatísticos basados en un mayor número de muestras que aquellas estudiadas en la primera contribución. Se analizan 60 muestras provenientes de 15 perfiles columnares. El recuento de palino- 
morfos se hizo hasta alcanzar 150 a 200 registros por muestra y en algunos casos esta cifra fue superada.

Para la preparación del material se siguió el método de Lee (1964) y la acetolisis. El montaje se hizo en gelatina-glicerina.

Las observaciones se hicieron con un microscopio Zeiss Standard WL. Las fotomicrografías fueron tomadas con la cámara del mismo microscopio usando película KODAK de 22 DIN.

Las preparaciones quedan depositadas en la Palinoteca del Departamento de Geología y Paleontología de la Universidad de Concepción.

Los palinomorfos escasamente representados y los que no se han podido determinar por su mal estado de convervación se incluyen en el item "indeterminados".

Para asociar los mantos entre sí, se escogió el método de correlación matemática que permite comparar cada manto con los otros cuatro sobre la base de los porcentajes de los distintos palinomorfos.

Este método consiste básicamente en la estimación del grado de asociación entre variables (mantos), el cual se expresa a través del coeficiente de correlación " $r$ ", el cual varía entre $1 \mathrm{y}-1$. Un valor " $r$ " igual a 0 indica una ausencia total de asociación. Se utiliza aquí el modo $\mathrm{Q}$ de operación ya que se correlacionan observaciones entre sí (porcentajes de palinomorfos) (Miller y Steven, 1962).

Los datos para obtener los coeficientes de correlación fueron procesados en un computador IBM 370.

\section{b) Descripción de los palinomorfos.}

Se deja constancia en este capítulo de los nuevos registros obtenidos, los que deben sumarse a los 25 tipos de palinomorfos descritos en la primera comunicación.

\section{PTERIDOPHYTA}

Género Cyathidites Couper 1953

Cyathidites australis Couper 1953 (Lám. I. Fig. 1)

Esporas triletes, biconvexas, de contorno ecuatorial triangular a subtriangular, de 50 a $80 \mu$ de diámetro ecuatorial, lados ligeramente cóncavos, ángulos ampliamente redondeados. Lesura de borde liso, no llega a tocar los ángulos. Exina psilada, de aproximadamente $2 \mu$ de grosor.

Esta especie es mucho menos frecuente que Cyathidites minor Couper 1953.

Se han observado, aunque poco frecuentes, una serie de esporas triletes y monoletes, las que no se han podido definir claramente por el momento. Para los efectos de conteo se hace referencia a ellas como Trilete y Monolete respectivamente.

Los triletes presentan exinas que van desde psiladas, a rugulado-verrucosas o tuberculadas.

\section{GYMNOSPERMAE}

Género Trisaccites Cookson y Pike 1954

(Lám. I. Fig. 2)

Granos trisacados. Cuerpo de contorno subtriangular a redondeado en vista polar, de 25 a $30 \mu$ de diámetro ecuatorial. Sacos pequeños, alargados, de 10 a $15 \mu$ de ancho y de 6 a $7 \mu$ de alto. Exina finamente granulosa o verrucada, de $\mathrm{m} / \mathrm{m} 1 \mu$ de grosor.

Se han encontrado muy pocos ejemplares. Es probable que se trate de Trisaccites microsaccatus Couper 1960.

\section{Género Podocarpidites Cookson ex Couper 1953}

Se han observado tres especies adscriptas a este género. En la primera contribución se hace una descripción a nivel genérico. Aquí se describen las especies por separado, sin atribuirles nombre por el momento. Se adopta la terminología para las medidas de los granos propuesta por Romero, 1978.

\section{Podocarpidites sp. 1. (Lám. I. Fig. 3)}

Granos bisacados. Cuerpo de contorno subcircular en vista polar (generalmente el largo es algo mayor que el ancho) y lentiforme en vista ecuatorial. Largo del cuerpo de 24 (35) $40 \mu$, ancho del cuerpo de 24 (30) $40 \mu$. Li mayoría de los granos están en vista polar o de forma tal que no se pudo medir con exactitud 
el alto del cuerpo, es de m/m $30 \mu$. Sacos de extremos redondeados, algo pequeños. Ancho del saco de 18 (25) $30 \mu$, alto del saco de 14 a $22 \mu$. Línea de inserción de los sacos de 15 a $20 \mu$ de largo, subparalelas. Largo total del grano de 32 (40) $50 \mu$.

Exina del cuerpo de 1 a $2 \mu$ de grosor, irregularmente ondulada o rugulada. Exina de los sacos muy semejante a la del cuerpo.

Es una forma muy cercana a $P$. otagoensis Couper 1953. Es la especie que se presenta con más frecuencia.

\section{Podocarpidites sp. 2 (Lám. I. Fig. 4)}

Granos bisacados. Cuerpo de contorno subcircular a elíptico en vista polar (generalmente el ancho es mayor que el largo). Largo del cuerpo de 25 (30) $52 \mu$, ancho del cuerpo de 35 (40) $65 \mu$. No se pudo medir el alto del cuerpo. Sacos semicirculares e elípticos, grandes. Ancho del saco de 25 (35) $67 \mu$, alto del saco de 15 (20) $42 \mu$. Línea de inserción de los sacos de 15 a $25 \mu$ de largo, paralelas a ligeramente curvas. Los sacos se contraen antes de llegar a la línea de inserción. Largo total del grano de $65(80) 100 \mu$.

Ornamentación del cuerpo punctitectada a finamente reticulada. Sacos reticulados. Retículo de los sacos rectimurado, angustimurado, heterobrochado, en partes fragmentimurado (los muri se interrumpen), más fino hacia los bordes. Lumina de 1 a $3 \mu$ de diámetro.

Es probable que se trate de $P$. marwickii Couper 1953, o alguna especie afín. Esta forma es menos frecuente que la especie anterior.

\section{Podocarpidites sp. 3}

(Lám. I. Fig. 5, 6 y 7)

Granos bisacados. Cuerpo de contorno circular a subcircular en vista polar. Largo del cuerpo de 55 a $65 \mu$, ancho del cuerpo de 50 a $58 \mu$. No se pudo medir el alto del cuerpo. Sacos semicirculares. Ancho del saco de 40 a $50 \mu$, alto del saco de 30 a $35 \mu$. Largo total del grano de 75 a $95 \mu$.

Exina del cuerpo de 2 a $2,2 \mu$ de grosor reticulada. Reticulo fino, curvimurado, angustimurado, homobrochado. Lumina de menos de $1 \mu$ de diámetro. Nexina poco notoria. Sacos notoriamente reticulados. Retículo rectimurado, angustimurado, heterobrochado, más fino hacia la línea de inserción de los sacos. Lumina de 1 a $3 \mu$ de diámetro, irregulares en forma. Muri delgados, de 0,2 a 0,5 $\mu$ de ancho.

Esta especie es poco frecuente y solamente se encontró en el manto 3.

Lamentablemente, no se ha encontrado en la literatura disponible especies a la cual se pueda adscribir esta forma.

\section{ANGIOSPERMAE DICOTYLEDONEAE \\ Género Proteacidites Cookson 1950 ex Couper 1953.}

Se han observado dos especies referidas a este género. Una de ellas, descrita en la primera contribución, es muy cercana a Proteacidites parvus Cookson 1950. La especie que se describe a continuación es menos frecuente, y entre las formas fósiles tiene afinidad con Proteacidites incurvatus Cookson 1950.

\section{Proteacidites sp. 2}

(Lám. I. Fig. 8 y 9)

Granos triporados, de contorno ecuatorial subtriangular, de 30 a $70 \mu$ de diámetro ecuatorial, lados cóncavos en vista polar. Poros ecuatoriales, circulares, cóncavos, de 4 a $5 \mu$ de diámetro. Zona de las aberturas protuberante. Exina finamente reticulada a punctitectada, de $\mathrm{m} / \mathrm{m}$ $3,5 \mu$ de grosor. Nexina poco notoria.

\section{Género Psilatricolporites (Hammen) ex Potonié 1966}

Granos tricolporoidados, prolatos a subprolatos, de 25 a $30 \mu$ de diámetro polar y 15 a $22 \mu$ de diámetro ecuatorial. Colpes de 15 a $20 \mu$ de largo. Ora bien notorios, circulares. Exina de aproximadamente $1 \mu$ de grosor, psilada.

$20 \mu$ de largo. Ora bien notorios, circulares. Exina de aproximadamente $1 \mu$ de grosor, psilada.

Son numerosas las Angiospermae que tienen granos con estas caracteristicas y pertencen a familias distintas, por lo cual no se puede dar la afinidad botánica de esta forma. 


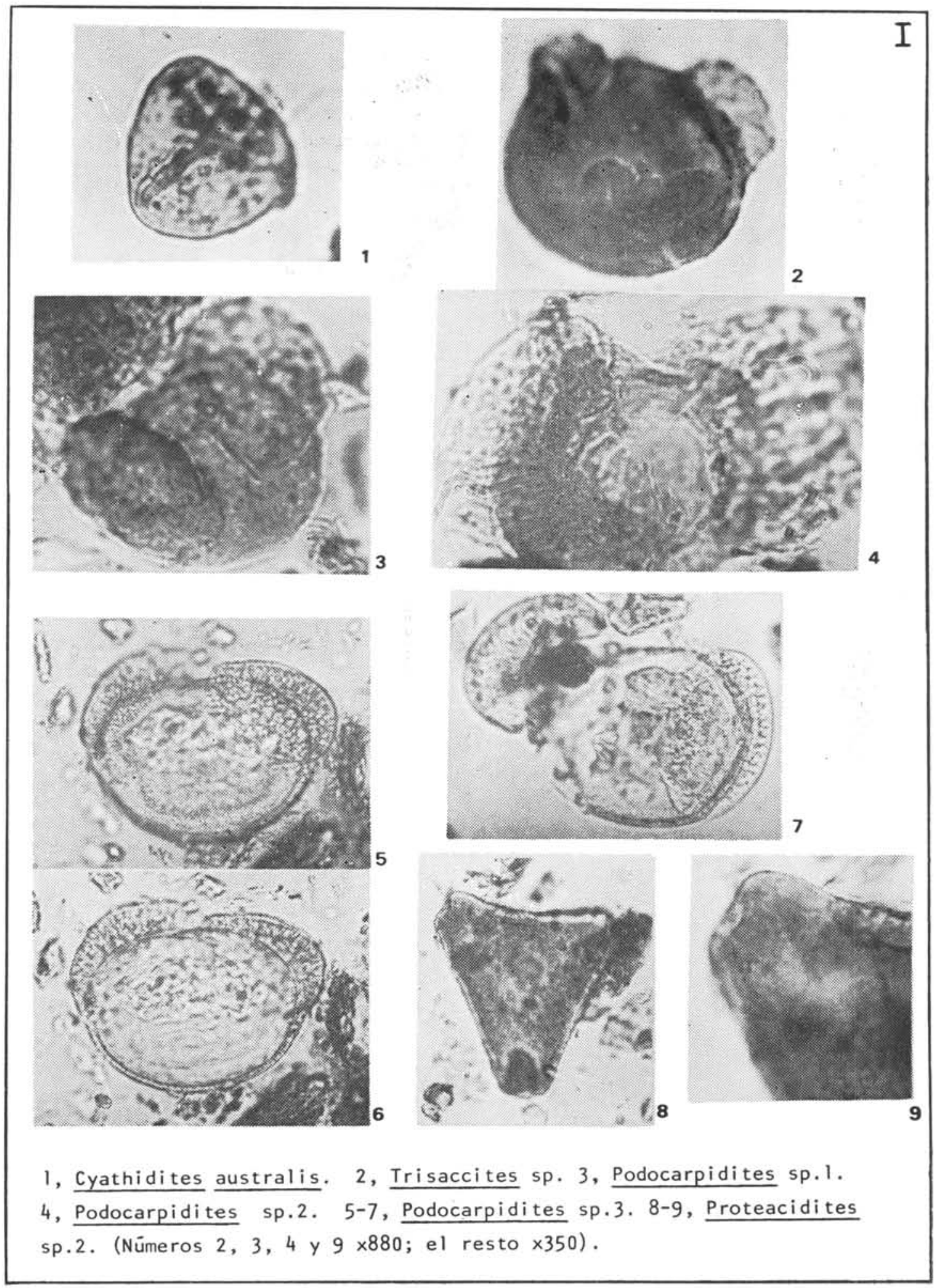




\section{Género Polyporina (Naumova) Potonié 1960.}

Granos poliporados, esféricos a subesféri$\cos$, de 25 a $30 \mu$ de diámetro. Poros subcircula. res, de contorno irregular, de $\mathrm{m} / \mathrm{m} 3 \mu$ de diámetro. Exina de 1 a $2 \mu$ de grosor. Sexina punc titectada.

Forma semejante a las actuales Caryophyllaceae. Entre las especies fósiles tiene afinidad con Caryophyllidites polyoratus Couper 1960, pero tiene menor número de poros que ésta. Polyporina romeroi Archangelsky 1973 es la forma más próxima entre los fósiles.

Palinomorfo poco frecuente.

Se han observado granos tricolporoidados y monoletes, escasamente representados, cuyas características no permiten incluirlos en los géneros descritos. Para los efectos de conteo se hace referencia a ellos como Tricolporites Erdtman 1947 y Monosulcites Cookson ex Couper 1953, respectivamente, ya que las descripciones dadas para estos géneros son las más apropiadas.

\section{RESULTADOS OBTENIDOS EN EL ANALISIS PALINOLOGICO}

Aunque se han detectado algunas leves variaciones verticales en algunos perfiles, éstas no se toman en cuenta en la elaboración de datos e interpretaciones por no ser significativas. Pero es interesante dejar constancia de ellas, tal es el caso de los Fungi que disminuyen algo de base a techo en el manto $4 \mathrm{y}$ de las Pteridophyta que aumentan en igual sentido en el mismo manto. Por otra parte en algunos perfiles de los mantos 2 y 3 las Dicotyledoneae aumentan muy poco de base a techo, y en algunos perfiles de los mantos 5 y 7 se observa un leve aumento de Pteridophyta de base a techo.

Por otra parte, los porcentajes de palinomorfos en los distintos perfiles de un mismo manto son muy semejantes. La variación horizontal tampoco es significativa.

Por lo tanto, para exponer los resultados y elaborar las interpretaciones se considera al manto como un todo.

\section{DISTRIBUCION DE LOS PALINOMORFOS EN LOS MANTOS}

En la tabla № 1, presentan para cada manto, los porcentajes de todos los palinomorfos determinados en ambas comunicaciones, ya que han variado las cifras debido a los nuevos registros 7 al mayor número de muestras analizadas.

\section{COMPARACION ENTRE LOS MANTOS}

Las relaciones existentes entre los mantos se analizaron ya en la primera comunicación, y los conceptos generales allí expuestos se han confirmado matemáticamente por medio del cálculo del coeficiente de correlación.

A continuaçión se presenta una matriz con los coeficientes de correlación calculados. Las variables corresponden al número de mantos en estudio (anotados como M2, M3, M4, M5 y M7). Se han tomado en cuenta 31 observaciones correspondientes a los porcentajes de cada tipo de palinomorfos (salvo los de posición sistemática incierta y aquellos indeterminados).

$\begin{array}{cccccc} & \text { M2 } & \text { M3 } & \text { M4 } & \text { M5 } & \text { M7 } \\ \text { M2 } & 1 & & & & \\ \text { M3 } & 0.99 & 1 & & & \\ \text { M4 } & 0.22 & 0.22 & 1 & & \\ \text { M5 } & 0.93 & 0.93 & 0.39 & 1 & \\ \text { M7 } & 0.88 & 0.89 & 0.57 & 0.94 & 1\end{array}$

Analizando el comportamiento de cada manto con respecto a los otros cuatro, se deduce que los resultados en cifras concuerdan plenamente con los conceptos básicos expuestos en el trabajo anterior.

También mantienen su vigencia y sin mayores modificaciones lo referente a las características de la paleoflora y a las deducciones paleoclimatológicas y paleoecológicas.

\section{CONCLUSIONES}

Con el trabajo realizado durante esta primera etapa de las investigaciones paleopalinológicas en el Terciario de Arauco-Concepción se ha logrado detectar aquellos tópicos donde es de interés profundizar y una visión global del 
Paleobotânica e Áreas Afins na América do Sul _ $\quad$ Bol. IG. Instituto de Geociências, USP, V. 11:31-189, 1980

TABLA N? 1

\begin{tabular}{|c|c|c|c|c|c|}
\hline & MANTO 2 & MANTO 3 & MANTO 4 & MANTO 5 & MANTO 7 \\
\hline Inapertisporites & 5.111 & 2.897 & 14.109 & 6.471 & 6.919 \\
\hline Monoporisporites & 0.320 & - & 2.877 & 2.345 & 0.624 \\
\hline Lacrimasporonites & 0.385 & 0.268 & 2.633 & - & 0.474 \\
\hline Dicellaesporites & 0.740 & 0.330 & 3.914 & - & 1.339 \\
\hline Multicellaesporites & 2.898 & 1.655 & 14.128 & 0.990 & 6.675 \\
\hline Pluricellaesporites & - & - & 2.014 & - & - \\
\hline Granatisporites & 0.391 & 0.235 & 3.606 & - & 0.697 \\
\hline TOTAL FUNGI & 9.84 & 5.39 & 43.28 & 9.81 & 16.73 \\
\hline Trilete sp. & 0.800 & 0.211 & 0.337 & 1.604 & 0.430 \\
\hline Gleicheniidites & 5.306 & 6.867 & 11.546 & 13.848 & 12.456 \\
\hline Clavifera & 3.983 & 5.204 & 3.744 & 2.165 & 7.140 \\
\hline Cyathidites minor & 6.592 & 9.044 & 11.542 & 11.092 & 11.895 \\
\hline Cyathidites australis & 0.614 & 1.849 & 0.823 & - & 0.249 \\
\hline Leiotriletes & 0.206 & 0.523 & 2.499 & - & 0.645 \\
\hline Monolete sp. & 0.197 & 0.430 & 0.122 & - & - \\
\hline Laevigatosporites & 0.452 & 0.854 & 7.849 & 2.812 & 1.870 \\
\hline Peromonolites & 0.196 & 0.248 & 0.397 & 0.477 & 0.520 \\
\hline TOTAL PTERIDOPHYTA & 18.35 & 25.23 & 38.86 & 32.00 & 35.20 \\
\hline Podocarpidites sp. 1 & 1.197 & 0.515 & 0.20 & 4.636 & 1.290 \\
\hline Podocarpidites sp. 2 & 0.550 & 0.350 & - & - & - \\
\hline Podocarpidites sp. 3 & - & 0.454 & - & - & - \\
\hline Trisaccites & 0.590 & - & - & - & - \\
\hline TOTAL GYMNOSPERMAE & 2.34 & 1.32 & 0.20 & 4.64 & 1.29 \\
\hline Haloragacidites harrisii & 42.819 & 38.524 & 4.313 & 31.364 & 23.416 \\
\hline Proteacidites sp. 1 & 5.917 & 6.471 & 0.751 & 3.670 & 4.576 \\
\hline Proteacidites sp. 2 & - & 0.250 & - & - & 0.123 \\
\hline Myrtaceidites & 5.171 & 5.521 & 3.198 & 2.880 & 5.205 \\
\hline Cupaneidites & 0.311 & 0.240 & 0.421 & 0.075 & 0.343 \\
\hline Retitricolporites & 3.954 & 1.799 & 0.956 & 5.670 & 2.936 \\
\hline Psilatricolporites & 0.757 & 1.006 & 0.512 & - & 0.495 \\
\hline Tricolporites & 0.868 & 0.620 & 0.610 & 0.827 & 1.281 \\
\hline Tricolpites & 0.300 & 0.603 & 0.712 & 1.188 & 0.558 \\
\hline Polyporina & - & 0.098 & - & - & 0.526 \\
\hline TOTAL DICOTYLEDONEAE & 60.10 & 55.13 & 11.47 & 45.67 & 39.46 \\
\hline Monosulcites sp. & 0.874 & 0.638 & 0.185 & - & 0.305 \\
\hline Psilamonocolpites & 1.869 & 3.299 & 0.165 & - & 0.881 \\
\hline TOTAL MONOCOTYLEDONEAE & 2.74 & 3.94 & 0.35 & - & 1.19 \\
\hline Sphagnum sp? & 0.04 & 0.05 & 0.91 & - & 0.28 \\
\hline Araucariacites sp.? & 0.28 & 1.02 & 2.30 & 1.25 & 0.97 \\
\hline Diporites & 2.72 & 1.49 & 1.26 & 1.37 & 2.97 \\
\hline Palinomorfo espinoso sp. 1 & 2.34 & 4.36 & 0.06 & 0.15 & - \\
\hline Palinomorfo espinoso sp. 2 & 0.66 & 1.00 & - & - & 0.19 \\
\hline Otros indeterminados & 0.610 & 1.07 & 1.49 & 5.11 & 1.74 \\
\hline TOTAL & 100. & 100. & 100. & 100. & 100. \\
\hline
\end{tabular}

Tabla 1 - Porcentajes de los palinomorfos para cada manto. 
problema, lo cual permitió trazar las líneas de investigación a seguir.

Los nuevos registros encontrados, y el mayor número de muestras analizadas no han cambiado sustancialmente las conclusiones a las cuales se llegó en la primera comunicación en lo que a la paleoflora se refiere. La variación se produce con respecto a las Gymnospermae; se han detectado tres especies de Podocarpidites y algunos granos de Trisaccites lo cual ha elevado en forma notoria la representación de este grupo.

Los palinomorfos a los cuales se ha hecho referencia como Diporites y "palinomorfos espinosos", son probablemente elementos del fitoplancton y están en estudio por el momento.

$\mathrm{La}$ paleoflora indica que probablemente durante el Terciario inferior la zona haya sido un ambiente pantanoso, costero, tranquilo, en el cual prevaleció un clima subtropical húmedo.

Sobre la base del contenido palinológico se pueden diferenciar en mayor o menor grado los distintos mantos. En cambio la variación horizontal y la vertical dentro de cada manto no son significativas.

Actualmente se analiza el perfil completo del Miembro Lota para completar el estudio con todas las rocas de la secuencia.

En un futuro inmediato se continuará con el análisis palinológico de formaciones más jóvenes para obtener el registro completo de la secuencia terciaria y una amplia visión del proceso evolutivo de la flora y por ende del clima y del ambiente durante el Terciario en la zona de Arauco-Concepción.

\section{BIBLIOGRAFIA}

LEE, H.W., 1964. A modified method of coal maceration and a simple technique for slide preparation. Micropaleontology. 10 (4): 486-490.

MILLER, R., \& STEVEN, J., 1962. Estatistical Analysis in the Geological Sciences: 1-483. John Wiley and Sons. New York, London.

MUÑOZ CRISTI, J., 1968. Contribución al conocimiento geologico de la región situada al sur de Arauco y participa. ción de material volcánico en los sedimentos eocenos. En: CECIONI, G. El Terciario de Chile, Zona Central. 63-93. Ed. Andrés Bello, Santiago.

PALMA-HELDT, S., 1978. Contribución al conocimiento palinologico de los mantos carbon tferos del Terciario de Arauco-Concepcion, Chile. Actas I Congreso Latinoamericano de Paleontología.

ROMERO, E.J., 1978. Polen de gimnospermas y fagáceas de la Formación Rio Turbio (Eoceno), Santa Cruz, Argentina.: 1-223, 12 lám. Fundación para la Educación, la Ciencia y la Cultura. Buenos Aires.

\section{PALINOLOGIA DE LOS SEDIMENTOS PLIOCENICOS Y CUATERNARIOS DE LA SABANA DE BOGOTA}

\section{HERNANDO DUENAS J.}

Estratigrafía, INGEOMINAS, Carrera 30 \# 51-59, Bogotá, D.E., Colombia.

\section{RESUMEN}

Con base en los estudios palinológicos de los sedimentos Plio-Pleistocénicos de la Formación Tilatá, se ha podido determinar un levantamiento suave y gradual de la Cordillera Oriental de los 